\title{
Heterogeneity of breakpoints in non-LCR-mediated large constitutional deletions of the 17q11.2 NF1 tumour suppressor region
}

\author{
H Kehrer-Sawatzki, S Tinschert, D E Jenne
}

J Med Genet 2003;000:e1 16 (http://www.jmedgenet.com/cgi/content/full/40/10/e1 16)

$\mathrm{N}$ eurofibromatosis type 1 (NFl) is caused by mutations of the NFl gene at $17 \mathrm{q} 11.2$. The encoded protein, termed neurofibromin, contains a Ras-GTPase activating (RasGAP) domain, which accelerates the inactivation of Ras. Homozygous inactivation of neurofibromin is associated with a dysregulation of Ras mediated signalling pathways and tumorigenesis in NFl patients. ${ }^{12}$ More than $70 \%$ of the germline mutations are intragenically distributed throughout the coding region and are protein truncating. ${ }^{3}{ }^{4}$ In $5-20 \%$ of all NF1 patients, however, heterozygous microdeletions at $17 q 11.2$ have been identified. ${ }^{5-8}$ These patients with microdeletions often have a severe clinical phenotype characterised by facial dysmorphy, excessive numbers of neurofibromas, and mental deficits. In the majority of cases, the deletions at $17 q 11.2$ span $1.4 \mathrm{mbp}$ and are caused by recombination between highly homologous low copy repeats (LCR), which flank the NFI gene at distances of $\sim 400 \mathrm{~kb}$ on the proximal side and $700 \mathrm{~kb}$ on the distal side. ${ }^{9-13}$ The LCRs mediating intrachomosomal deletions at $17 q 11.2$ are derived from the $W I-12393$ gene and contain sequences with high homology to 19p13. ${ }^{11} 1314$ The structure of the NF1 gene region at $17 \mathrm{q} 11.2$ is further complicated by other duplicated sequences, such as the pseudogene fragments of the SMURF2 and the KIAA0160 genes located at 17q. ${ }^{14}$ These sequences may also represent templates for unequal recombinations and subsequent deletions of the intervening chromosomal regions at 17q11.2. Indeed, five patients with large deletions in $17 q$ spanning more than 1.4 mbp have been described. ${ }^{5} 9^{10}{ }^{15-17} \mathrm{Up}$ to now, breakpoints in these patients with long range deletions have not been placed precisely within the physical map of chromosome 17q11-12, which is still uncertain for a few regions.

In this study, we determined the precise boundaries of the constitutional deletion in patient BUD who was first reported by Jenne et al. ${ }^{10}$ The deletion encompasses the commonly deleted $1.4 \mathrm{mbp}$ interval, and additional $3.3 \mathrm{mbp}$ in a distal direction. We established the complete physical map and gene content data for the entire deletion of patient BUD distal to the WI-12393 gene, and identified the orthologues for all human genes in the mouse genome. In the genomic interval telomeric to the distal NFl LCR, conserved gene order is observed when comparing human and mouse except for the schlafen $(S L F N)$ gene cluster, which is partially lost in the human genome. Hence the distal break in this patient occurred in a genomic region which has experienced several local rearrangements during primate and rodent evolution.

\section{METHODS}

\section{Patient BUD}

The male 18 year old patient BUD was the first born child of dizygotic twins. Delivery was by caesarean section at 37 weeks' gestation. His birth weight was $2300 \mathrm{~g}$, length $46 \mathrm{~cm}$, and head circumference $32 \mathrm{~cm}$. Neurofibromatosis was

\section{Key points}

- Deletions of the entire neurofibromatosis type 1 (NF1) region at 17 q11.2 most often span the same $1.4 \mathrm{mbp}$ interval and are caused by meiotic recombinations between low copy repeats (LCR) which flank the deleted interval. Several NF1 patients with even larger deletions at $17 q$ have been reported, but the position of their breakpoints within a contiguous BAC/PAC contig has not been determined.

- The molecular characterisation of a deletion spanning more than $1.4 \mathrm{mbp}$ in NF1 patient BUD is described and compared with that in four patients who have previously been characterised by marker analysis and Southern hybridisation using short genomic probes (D17S117, D17S120, D17S57, D17S73, D17S115). Both the proximal and distal breakpoints of all five patients clearly fall at different locations and are not bordered by LCRs. The centromeric break in patient BUD was mapped to BAC $271 \mathrm{~K} 11$ at $17 \mathrm{q} 11.2$ between a partial SMS repeat and the WI-12393 derived LCR, whereas the distal break was located between the two SCHLAFEN (SLFN) genes, SLFN1 and SLFN3, at $17 \mathrm{q} 12$.

- Comparisons between the otherwise conserved human and mouse segments showed major differences in the number and orientation of SLFN genes. Thus the distal breakpoint of patient BUD lies in a region containing multiple evolutionary breakpoints. The deletion was shown to be paternally inherited and to occur through an intrachromosomal mechanism. As three of four previously analysed NFI deletions that were not bordered by LCRs were also found to be of paternal origin, non-LCR-triggered deletions are most probably mitotic events during spermatogenesis.

- Patient BUD as well as patient UWA106-3, who also has a large deletion extending beyond the distal NFI LCR at 17q11.2, suffer from multiple spinal neurofibromas. Their deletions may include a modifier locus which predisposes these patients to the development of these tumours.

suspected shortly after birth, based on multiple café-au-lait spots. There was no family history of NFl.

All developmental milestones were delayed. He walked alone at five years. At age 13.8 years cognitive development was assessed at an eight year level. There were difficulties in gross and fine motor coordination. 
At the age of 14, magnetic resonance imaging (MRI) of the brain showed several hyperintense T2 weighted lesions in the left and right lentiform nucleus and internal capsule on both sides. The spinal MRIs showed multiple neurofibromas affecting the nerve roots on both sides in nearly all segments of the vertebral column, often filling the neural foramina. At levels $\mathrm{Cl} / 2$ to $\mathrm{C} 4 / 5$ and at the lumbosacral junctions, the lesions extended into the spinal canal, forming dumbbell tumours. Intraspinal tumours were present at C2/3 and C3/4. Multiple neurofibromas affecting the lumbosacral plexus were observed on both sides.

Physical examination at the age of 18 revealed 15 café-aulait spots, skinfold freckling, and more than a thousand cutaneous neurofibromas. Height $(162 \mathrm{~cm})$ and head circumference $(54 \mathrm{~cm})$ were below the third centile. There was a thoracic scoliosis, genu valgum, deformity of the feet, and hyperextensible joints. A large fleshy nose gave his face a coarse appearance. Hands $(19 \mathrm{~cm}$ ) and feet (European shoe size $38^{1 / 2}$ ) were not enlarged.

\section{FISH analysis}

Chromosome spreads were prepared from lymphocytes and EBV transformed lymphoblastoid cells from the patient, and from blood lymphocytes from his father, using standard methods. BAC clones were purchased from the BACPAC Resource Center (www.chori.org/bacpac). Cloned polymerase chain reaction (PCR) products and BAC DNA used as fluorescent in situ hybridisation (FISH) probes were labelled with biotin-16-dUTP (Roche Diagnostics, Mannheim, Germany) and detected with FITC-avidin and biotinylated anti-avidin (Vector Inc, Burlingame, California, USA) or labelled with digoxygenin-11-dUTP (Roche Diagnostics) and detected by mouse anti-digoxygenin antibodies, and in a second step with anti-mouse antibody conjugated with Texas-Red (Dianova, Hamburg, Germany). Slides were counterstained with diamidinophenylindole (DAPI) and mounted with Vectashield antifade solution (Vector Inc).

\section{PCR products used as FISH probes to narrow the deletion boundaries}

PCR products were amplified from BAC DNA using the Expand Long-Template PCR system (Roche Diagnostics) and the primers listed in table 1 . The PCR products were purified by $\mathrm{GFX}^{\mathrm{TM}}$ DNA purification columns (Amersham Pharmacia, Freiburg, Germany) and cloned using the TOPO-TA cloning kit (Invitrogen, Karlsruhe, Germany). The authenticity of recombinant clones was confirmed by sequencing or restriction enzyme digestion. DNA was prepared from the cloned PCR products with the MIDI DNA isolation kit (Qiagen, address) and labelled by nick translation.

\section{BAC library screening to complete the map of the $17 q 11.2-12$ region}

Filters from the RPCI-11 human BAC library and the RPC 1, 3 , and 5 human PAC libraries were obtained from the German Resource Center (www.rzpd.de) and the BACPAC Resource Center (RZPD) (www.chori.org/bacpac). Screening of the libraries was done by hybridisation with markers amplified by PCR and labelled by random priming with hexamer oligonucleotides and Klenow polymerase in the presence of ${ }^{32} \mathrm{P}$-dCTP (Amersham Pharmacia) in buffer containing $7 \%$ SDS, $0.5 \mathrm{M}$ Na phosphate $(\mathrm{pH} 7.2)$, and $1 \mathrm{mM}$ EDTA at $65^{\circ} \mathrm{C}$ overnight. The positions of these markers are indicated in fig 3 and the primer sequences are available from the authors by request. Filters were washed four times at increasing stringency, with a final wash of $0.2 \times \mathrm{SSC} / 0.1 \%$ SDS for 45 minutes. Following autoradiography for one to two days at $-70^{\circ} \mathrm{C}$ with intensifying screens, positive clones were identified by $\mathrm{x} / \mathrm{y}$ coordinates and obtained from BACPAC resources or the RZPD.

\section{Genotyping}

To determine both the parental origin of the deletion and the recombination mechanism underlying this rearrangement, marker haplotypes were reconstructed and the segregation of marker genotypes was investigated using DNA isolated from peripheral blood lymphocytes of patient BUD and his family members. Ten polymorphic microsatellite markers were analysed, five proximal (D17S841, D17S1873, D17S1841, D17S975, D17S1294) and four distal to the deletion boundaries of patient BUD (D17S907, D17S1833, D17S1788, D17S1867). Marker GGAA7D1l is located within the deleted region. Oligonucleotide primer sequences were obtained from the Genome Database and the respective forward primer was

Table 1 Cloned polymerase chain reaction products used as fluorescent in situ hybridisation (FISH) probes to confine the deletion breakpoints of patient BUD

\begin{tabular}{|c|c|c|c|c|c|}
\hline $\begin{array}{l}\text { PCR } \\
\text { primer }\end{array}$ & Sequence $\left(5^{\prime} \rightarrow 3^{\prime}\right)$ & $\begin{array}{l}\text { BAC (GenBank } \\
\text { accession } \\
\text { number) }\end{array}$ & $\begin{array}{l}\text { PCR product } \\
\text { (size in bp) }\end{array}$ & Position* & $\begin{array}{l}\text { FISH } \\
\text { results }\end{array}$ \\
\hline DJ2384 & GGATGCAAACAGGGAGATTIT & $271 K 11$ & DJ2384/2385 & $10862-$ & nd \\
\hline DJ2385 & GTGATGCAGG GAAGGAAAAAC & (AC005562) & (7067) & 17929 & \\
\hline DJ2386 & GGTGGCCAGTGAGGATAACAC & $271 \mathrm{~K} 11$ & DJ2386/2387 & $30519-$ & nd \\
\hline DJ2387 & CCCAGAAGGTGACTCAGGAAG & (AC005562) & $(7457)$ & 37976 & \\
\hline DJ2447 & GGGGGAGAACGAATGTCCAG & $271 \mathrm{~K} 11$ & DJ2447/2448 & $40-901-$ & nd \\
\hline DJ2448 & CAGGCCTACTGCTGTGCTGTT & & (8320) & 49221 & \\
\hline DJ2516 & CTGATGGCATCCTGATTTGA & $271 \mathrm{~K} 11$ & DJ2516/2517 & $81044-$ & D \\
\hline DJ2517 & GAACAGCAGATTCACACAAGAGC & & $(7230)$ & 88274 & \\
\hline DJ2439 & TCGTGGATTATTGCCCTTCCT & 47L3 & DJ2438/2439 & $87476-$ & D \\
\hline DJ2438 & AAGACACAGCCCCCTTCATTG & (AC022706) & (8159) & 95635 & \\
\hline DJ2522 & СТCTIATTATGGAGTGGGGAGCAG & 686D22 & DJ2522/2523 & 163300 & D \\
\hline DJ2523 & TCTGATCATTAGGCGAAAGACAAT & (AC060766) & (6052) & 169352 & \\
\hline DJ2725 & CATGGTACAGGATGAGGAGTIT & $686 \mathrm{D} 22$ & DJ2725/2726 & $26002-$ & nd \\
\hline DJ2726 & CGAAGTCTTGTAATCCAACCTG & & $(7300)$ & 33302 & \\
\hline DJ2789 & GGAAAAGCAAGATTCAAATAGTAT & 686D22 & DJ2789/2788 & $10074-$ & nd \\
\hline DJ2788 & ATTCTGGTCTTAAATACAATCTCC & & $(5081)$ & 15155 & \\
\hline DJ2781 & CTITTCTGCCAGTCTITCTCC & 1094M14 & DJ2780/2781 & $20271-$ & nd \\
\hline DJ2780 & CAGACCCTTATTCTCCTGCTT & (AC015911) & $(6021)$ & 26292 & \\
\hline DJ2730 & AGTCTCTCCTTTCСССАСТTA & $1094 \mathrm{Ml} 4$ & DJ2729/2730 & 34230 & nd \\
\hline DJ2729 & TACAATGGGTTGAGGAATGA & (AC015911) & $(5620)$ & 39859 & \\
\hline
\end{tabular}

*Position of the PCR product on the respective BAC clone with respect to its accession number.

$D$, deleted; nd, not deleted; PCR, polymerase chain reaction. 
5' end-labelled with 6-FAM (Applied Biosystems, Foster City, California, USA). PCR products were analysed by electrophoresis on $5 \%$ denaturing polyacrylamide gels on a $396 \mathrm{ABI}$ DNA sequencer using the GENESCAN (version 2.1) software (Applied Biosystems). We phased the parental haplotypes on the basis of the most parsimonious explanation of the observed genotypes.

\section{RESULTS}

Characterisation of the proximal deletion breakpoint In a previous study, we showed by FISH analysis that BAC R$271 \mathrm{~K} 11$ hybridised to both chromosome 17 homologues in the majority of $\mathrm{NFI}$ deletion patients, but did not produce a second signal on metaphase chromosomes of the patient BUD. The probe R-271Klls, which spans the region of BAC
R-271K11 from 141950 to 148526 (according to AC005562), was clearly deleted in patient BUD, but present in the other deletion patients. ${ }^{10}$ To locate the proximal end of the deletion in patient BUD precisely, we narrowed down the deletion boundary with additional BACs (fig 1) from the 17q11.2 region. In contrast to the more proximal BAC R-281MIl, the BACs R-778K9 and 2349P2 1 were completely missing on one chromosome 17. We now found a highly reduced hybridisation signal with BAC R-271K11 on the second chromosome, indicating that it was not completely deleted and that some sequences from its insert still hybridised to the affected chromosome 17. Three subfragments from the proximal portion of the BAC insert were used in FISH experiments (table 1). For the PCR probes DJ2384/2385, DJ2386/2387, and DJ2447/2448, signals on both chromosomes were noted

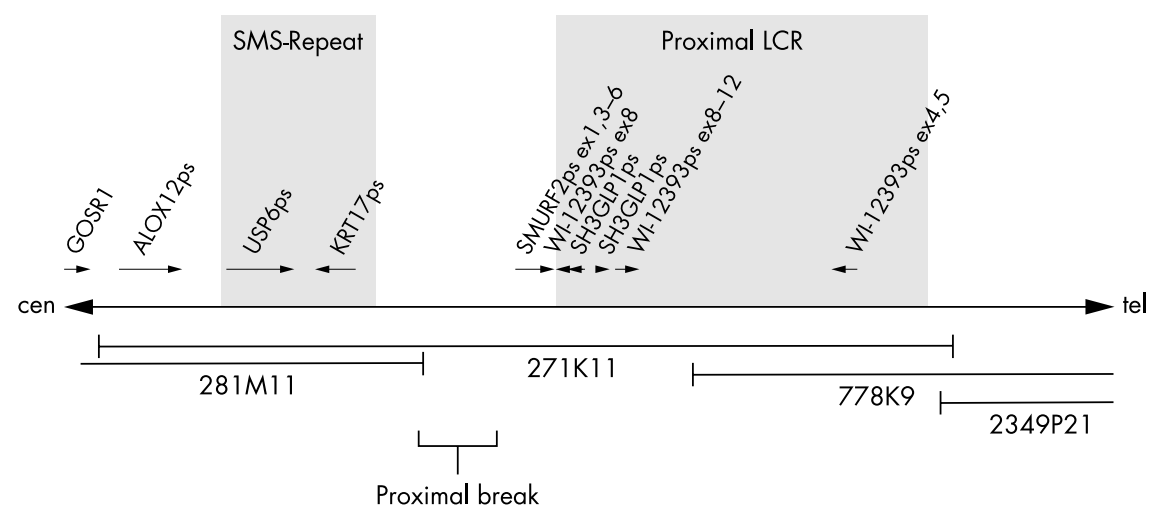

Figure 1 (A) Schematic map of the proximal deletion breakpoint region of patient BUD. The horizontal bars represent the position of BACs R-271K 11 (AC005562), R-281M11 (AC01 1840), R-778K9 (AC023266), and 2349P21 (AC127024). Horizontal arrows indicate the transcriptional direction of the genes and pseudogenes. The low copy repeats (LCR) mapped to this region, a partial SMS (Smith-Magenis-Syndrome) repeat and the proximal WI12393 containing LCR, are marked by grey rectangles.
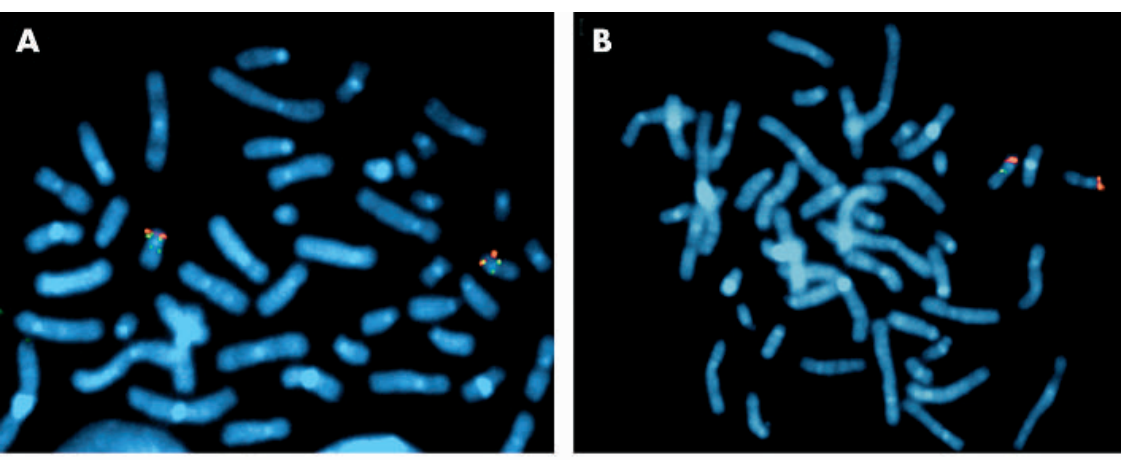

Figure 2 Confinement of the proximal (panels A and B) and distal (panels C and D) deletion breakpoints of patient BUD by fluorescent in situ hybridisation (FISH) with cloned polymerase chain reaction products (green) and, as control, BAC 1D5 (red), which maps to $17 p 13$ (panels A, B, and D). (A) Hybridisation of probe DJ2447/2448 (green) at 17g11.2 on both homologous chromosomes. This probe also hybridised to the short arm of chromosome 17. (B) FISH analysis with probe DJ2516/2517 (green) showed a deletion of the corresponding segment of one chromosome 17. (C) FISH with probe DJ2522/2523 (green) resulted
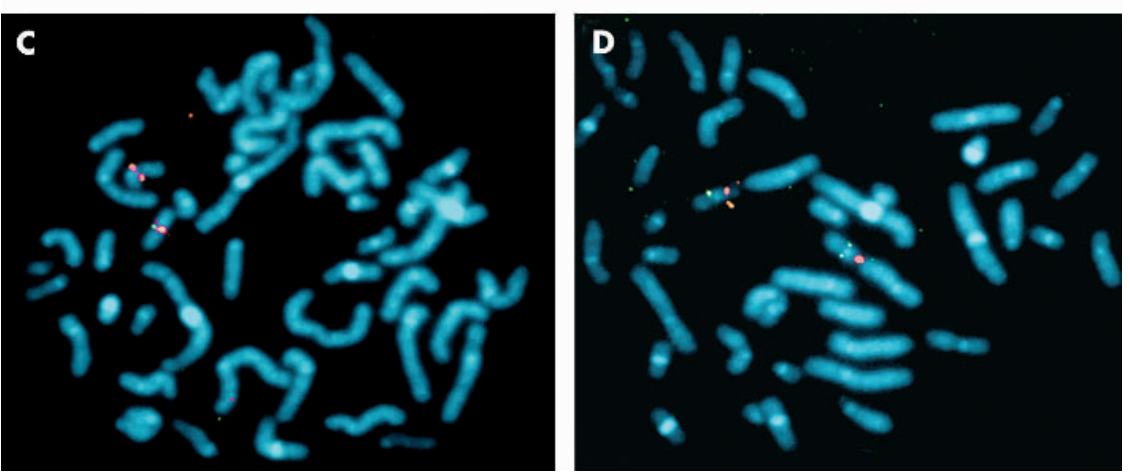
in signals only on one chromosome 17 , indicating that the corresponding region is deleted. Co-hybridisation was done with BAC 1094M14 (red), hybridising to both chromosomes 17. (D) Hybridisation of probe DJ2788/ 2789 is detected on both chromosomes 17 and thus marks the distal deletion boundary. 

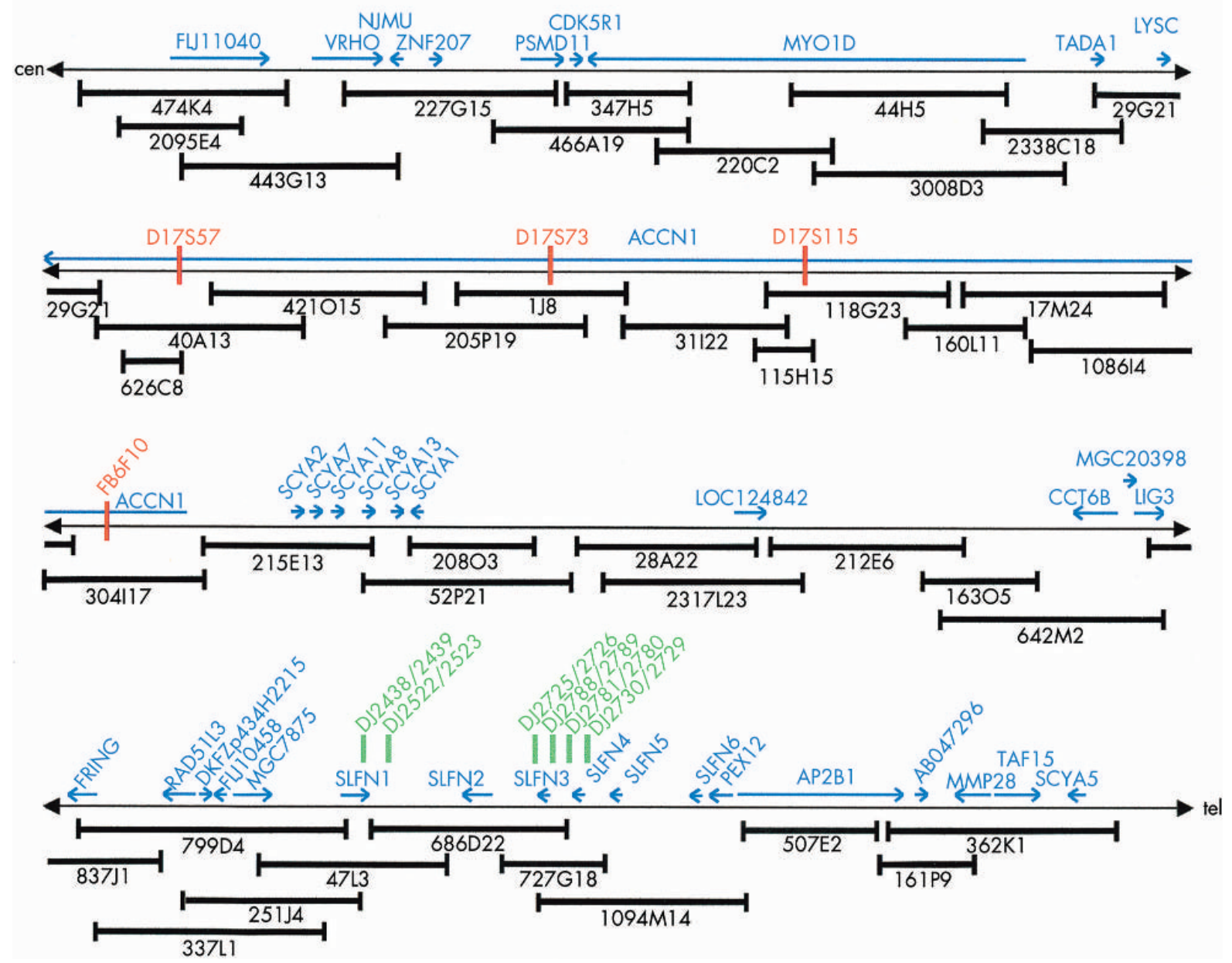

Figure 3 Schematic map of the 17q11.2-12 region from the FU11040 gene, which flanks the distal LCR in the NF1 gene region at 17q11.2 up to SCYA5 gene. The BAC/PAC clones which make up this contig are indicated by horizontal bars. The position and orientation of the genes mapped within this region are given by horizontal arrows in blue. The location of the FISH probes (DJ2438/2439-DJ2730/2729, in green) used to confine the distal deletion boundary of patient BUD are given, as well as those markers (red) that were previously used to investigate the deletion boundaries in other NF1 patients with large deletions.

(fig 2A), whereas probe DJ2516/2517 was clearly hybridising to only one chromosome 17 homologue (fig 2B). According to the position of the PCR primer pairs on BAC R-271K1l, the proximal break occurred between 49221 and 81044 (numbering according to GenBank entry AC005562 of BAC R-271K11; table 1). Thus the proximal breakpoint of BUD lies far outside of the LCR interval (112000 and 147000 on R-271K11) which recurs on BAC R-640N20, on the distal side of the NFI gene. ${ }^{911} 13$

\section{Identification of the distal breakpoint}

To confine the distal end of the deletion, we assembled a BAC contig for the $3.3 \mathrm{mbp}$ distal to the WI-12393 related LCR on BACs R-640N20 and R-474K4. This contig covers the previously identified clones ${ }^{18}$ and BAC R-337Ll, ${ }^{10}$ and ends up with BAC R-362Kl, as shown in fig 3.

Overlapping clones were identified by BLAST searches using completely sequenced BAC clones, BAC end sequence, and clone pools which were screened with known STS markers from the existing radiation hybrid (Standford, Genebridge 4) and genetic linkage maps (Marshfield genetic map). This assembly was confirmed by FISH analyses with several BAC clones from this region on metaphase chromosomes from different deletion patients. The deletion of patient BUD extends up to BAC R-799D4, which is the most distal clone that is completely deleted on the affected chromosome 17. Interphase nuclei of peripheral blood leucocytes were also investigated by FISH with BAC R799D4 and a differentially labelled BAC R-55Al3 (AC015651), which maps outside of the deleted region at 17q23. The interphase nuclei of patient BUD always lacked the second signal for R-799D4 $(\mathrm{n}=200)$, but displayed two signals for R-55Al3. By contrast, FISH analysis with BAC R$686 \mathrm{D} 22$ resulted in two signals of unequal intensity on chromosome 17 homologues, indicating that this BAC is hitting the distal breakpoint region. BAC R-1094M14, which starts in the middle of R-686D22 and extends beyond its distal half, hybridised equally well to both chromosomes 17 . In order to restrict the distal deletion boundary more precisely, we undertook FISH with cloned PCR products DJ2438/2439 and DJ2522/2523 (table 1; fig 3). Both probes are deleted on one chromosome 17 of patient BUD (fig 2C). Subsequently, we generated probes DJ2788/2789 (fig 2D), DJ2725/2726, DJ2781/2780, and DJ2730/2729, which hybridised to both chromosomes of patient BUD and thus delineate the distal border of the deletion. According to these observations, the distal deletion breakpoint is located in the region between the SLFNI and the SLFN3 genes (fig 3). 
Characterisation of the deleted interval in patient BUD and comparison with the mouse syntenic region on chromosome 11

The deletion of patient BUD encompasses $4.7 \mathrm{mbp}$ including the 14 known genes between the proximal and distal NFI LCR at 17q11.2-CYTOR4, FLJ12735, FLJ22729, CENT2A, MGC13061, NF1, OMG, EVI2B, EVI2A, KIAA1821, MGC11316, HCA66, KIAA0160, and WI-12393-and 27 additional genes located distally to this region, ranging from FLJ11040 to the SCHLAFEN (SLFN) gene cluster (fig 3; table 2).

Comparative analysis of the NFI gene region in human and mouse undertaken by us previously ${ }^{14}$ showed that 12 of the 14 functional genes in the NFI gene region flanked by LCRs were also physically linked in the mouse genome on chromosome 11, but their order differed significantly. ${ }^{14}$ The subsequent region-beginning with the FLJ11040 gene and ending with the $S L F N$ gene cluster at the distal breakpoint region of patient BUD-is, however, strictly conserved with regard to the number and arrangement of genes in both species (table 2 ). In the region around the distal deletion boundary of patient BUD, the human and the mouse genomes again displayed significant discrepancies. Comparing the SLFN genes that are bordered by the MGC7875 and PEXI2 single copy orthologues, we noticed only six human members (SLFN1-6) of the SLFN gene family, but 10 murine genes in the otherwise conserved human-mouse interval (fig 4).

\section{Comparison with other long range deletions of $17 q$}

Through the last decade, three NFl patients with large deletions at $17 \mathrm{ql} 1-12^{591516}$ and one patient with a $7 \mathrm{mbp}$ deletion at $17 \mathrm{q} 11.2-21^{17}$ have been reported. The markers and FISH probes used in these studies have been identified by their sequence and placed on our physical map accordingly (fig 5). The proximal deletion boundary of patient UWA106-3

Table 2 Summary of the functional genes at 17q12 telomeric to the distal WI-12393 derived low copy repeats (LCR) and the mouse orthologues on chromosome 11 (11B5)

\begin{tabular}{|c|c|c|c|c|}
\hline $\begin{array}{l}\text { Human gene } \\
\text { (locus) }\end{array}$ & Accession No of the human cDNA & Mouse gene (locus) & $\begin{array}{l}\text { Accession No of the murine } \\
\text { orthologue }\end{array}$ & Features of the encoded protein \\
\hline FU11040 & NM_018307; AJ496730; AL136929 & Ak019059 & AK019059 & $\begin{array}{l}\text { ATP/GTP binding site motif } \mathrm{A}, \mathrm{Ca} \\
\text { binding EF hand }\end{array}$ \\
\hline VRHO & NM_138328; AJ313480 & Rhbd14 & NM_139228, AJ313479 & $\begin{array}{l}\text { Rhomboid-like, MT serine type } \\
\text { peptidase, EF hand }\end{array}$ \\
\hline NJMU & NM_022344; AF305686 & $A k 017667$ & AK017667 & $\begin{array}{l}\text { Highly expressed in fetal and adult } \\
\text { testis; spermatogenesis }\end{array}$ \\
\hline ZNF207 & NM_003457 & $Z f p 207$ & NM_011751; AB013357 & $\begin{array}{l}\mathrm{C} 2 \mathrm{H} 2 \mathrm{Zn} \text { finger, proline-rich, bipartite } \\
\mathrm{NLS}\end{array}$ \\
\hline PSMDI1 & NM_002815; ABO03102; AF001212 & Psmd 11 & AK012951; AK007547 & $\begin{array}{l}26 \mathrm{~S} \text { proteasome non-ATPase } \\
\text { regulatory subunit } 11\end{array}$ \\
\hline CDK5R1 & NM_003885; X80343; BC020580 & $C d k 5 r$ & NM_009871; U50707; S82819 & $\begin{array}{l}\text { Cyclin dependent kinase } 5 \text { activator } 1 \text {; } \\
\text { tau protein kinase II regulatory subunit }\end{array}$ \\
\hline MYOID & $X M \_126459 ; A B 018270$ & MyolD & XM_126459, BC039700 & Unconventional myosin \\
\hline TADA 1 & NM_015544; AL1 17619; AF132000 & Ak018332 & AKO18332, BCO11208 & Maid-like protein \\
\hline LYSC & XM_058864 & Ak006357 & AK006357 & Lysozyme C \\
\hline ACCN1 & NM_001094; U50352; U53212 & Accn 1 & NM_007384, AF348465, Y14634 & $\mathrm{ENaC}$ related, degenerin related \\
\hline SCYA2 & NM_002982 & Scya2 & NM_011333 & Member of SCYA family \\
\hline SCYAT & NM_006273 & Scya7 & NM_013654 & Member of SCYA family \\
\hline SCYA11 & NM_005408 & Scyall & NM_011330 & Member of SCYA family \\
\hline SCAY8 & NM_005623 & Scya8 & NM_021443 & Member of SCYA family \\
\hline SCYA13 & NM_005408 & Scyal2 & NM_011331 & Member of SCYA family \\
\hline SCYAl & NM_002981 & Scyal & NM_011329 & Member of SCYA family \\
\hline LOC124842 & XM_064333; BN000149; BC020591 & Loc217004 & XM_194796; BN000151 & Similar to KIAA1583 \\
\hline ССТ6B & NM_006584; D78333 & Cct6b & NM_009839 & $\begin{array}{l}\text { TCP20, CCT6B, T complex protein } 1 \text {, } \\
\text { zeta } 2 \text {, subunit } 6 \mathrm{~B} \text {, testis specific }\end{array}$ \\
\hline MGC20398 & XM_039437; BC11584 & $2410003 C 20 R i k$ & NM_025884 & $\begin{array}{l}\text { Neurofilament triplet } M \text { protein (weak) } \\
\mathrm{C} 2 \mathrm{H} 2 \text { type, NLS }\end{array}$ \\
\hline LIG3 & NM_002311; NM_013975 & Lig3 & NM_010716 & Ligase, testis specific splice form \\
\hline FRING & NM_057178 & Fring & NM_026097 & Zn finger; $\mathrm{C} 3 \mathrm{HC} 4$ type, testis specific \\
\hline RAD5IL3 & $\begin{array}{l}\text { NM_002878; NM_133628; } \\
\text { NM_133629 }\end{array}$ & $\operatorname{Rad} 51 / 3$ & NM_0111235 & $\begin{array}{l}\text { DNA repair protein, } \operatorname{RecA} \text {; ATP, GTP } \\
\text { binding site motif } A \text { (P loop) }\end{array}$ \\
\hline DKFZp434H2215 & NM_017559; BC024002 & Ak019639 & AK019639 & Containing FNIII domain \\
\hline FU10458 & NM_018096; AK001320 & Loc217011 & NM_145431; BC018399 & $\begin{array}{l}\text { G protein } \beta \text {; transducin-like; WD40 } \\
\text { repeat }\end{array}$ \\
\hline $\begin{array}{l}\text { MGC7875 } \\
\text { (unc }-45 \text { related) }\end{array}$ & AL832355; AL833281; XM_091530 & Loc217012 & XM_126446 & $\begin{array}{l}\text { Contains two armadillo repeats and a } \\
\text { TPR domain }\end{array}$ \\
\hline SLFNI & $\begin{array}{l}\text { NM_144975; BCO21238; AL832814; } \\
\text { AK075116; AK054668; }\end{array}$ & Slfn7 & AK036486 & \\
\hline SLFN2 & $\begin{array}{l}\text { NM_152270; AL512731; AK074184; } \\
\text { AK092241; AL831964 }\end{array}$ & Slfn8 & $\begin{array}{l}\text { NM_172796; XM_204663; } \\
\text { AK036579;AK050355 }\end{array}$ & \\
\hline SLFN3 & NM_018042; AK001122; BC035605 & Slfng & XM_204664;XM_137765 & \\
\hline SLFN4 & $\begin{array}{l}\text { NM_144682; AK074465; AK056514; } \\
\text { AL833747; AL832726 }\end{array}$ & Slfnio & XM_204665; XM_137766 & \\
\hline \multirow[t]{6}{*}{ SLFN5 } & BN000147 & & & \\
\hline & No equivalent & Slfn2 & NM_011408; AF099973 & $P$ loop (ATP/GTP binding site motif $A$ ) \\
\hline & No equivalent & Slfnl & NM_011407;AF099972 & $P$ loop (ATP/GTP binding site motif $A$ ) \\
\hline & No equivalent & Slfn4 & $\begin{array}{l}\text { XM_203494; XM_126110 } \\
\text { AF099976; AF099975; }\end{array}$ & $P$ loop (ATP/GTP binding site motif A) \\
\hline & No equivalent & Slfn3 & NM_011409, AF099974 & P loop (ATP/GTP binding site motif $A$ ) \\
\hline & No equivalent & Slfn5 & XM_204667; XM_137770 & \\
\hline SLFN6 & XM_064300 & Slfn6 & XM_137771, XM1̄11241 & \\
\hline PEX12 & NM_000286; U91521; AB004546 & Pex12 & BCO21800; NM_134025 & Peroxisome assembly protein 12 \\
\hline
\end{tabular}


Human

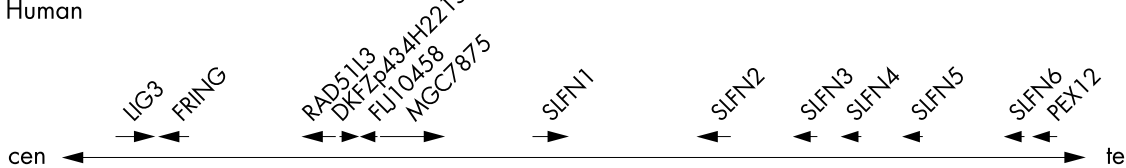

Figure 4 Comparative map of the region surrounding the distal deletion boundary of patient BUD in human and mouse. The length and the

transcriptional orientation of the respective genes are indicated by

Mouse

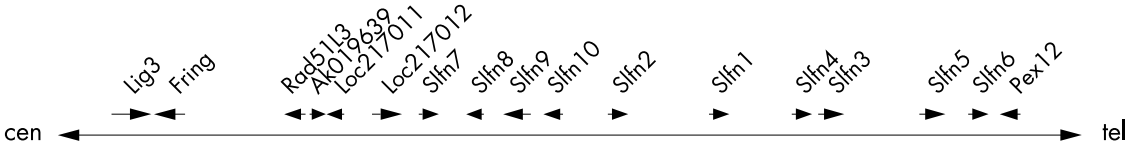

described by Kayes et al ${ }^{15}{ }^{16}$ was localised between D17S1294 and the SLC6A4 gene, ${ }^{19}$ the distal deletion breakpoint to the interval between the markers D17S73 and FB6F10 (figs 3 and 4). The proximal breakpoint of patient UWA155-1 described by Dorschner et al ${ }^{9}$ was located within the LCR bordered NFI interval between markers SHGC35088 and FB12A2, the distal deletion breakpoint between the markers D17S1656 and stSG50857 telomeric to the LCR flanking the 1.4 mbp region. ${ }^{9}$ The proximal breakpoint of the deletion of patient ID806 ${ }^{17}$ was located in the interval between marker D17S58 and the CRYBAl (Crystallin $\beta \mathrm{Al}$ ) gene. Thus the proximal breaks of the five patients clearly differ in their location. The distal breakpoints of these patients, however, have not been determined precisely. In three patients-UWA106-3, UWA155-1, and patient $3724 \mathrm{~A}^{5}$ - the deletion ends in the very large ACCNI gene which spans more than $1 \mathrm{mb}$, including the four markers D17S57, D17S73, D17S115, and FB6F10 (figs 3 and 5). The deletion reported by Upadhyaya et al ${ }^{17}$ is cytogenetically visible and is thus the largest. It ends somewhere between D17S73 and the RARA gene. The distal deletion breakpoint of our patient BUD is telomeric to the ACCNI gene in the region between the SLFN1 and SLFN3 genes. Blast searches with the entire WI-12393 gene sequence did not retrieve additional LCR copies in the region between D17S58 and the SLFN genes.

\section{Mechanism underlying the deletion}

We analysed several polymorphic markers on $17 \mathrm{q}$ in the parents and two brothers of the index patient. Lack of inheritance of the paternal allele for the GGAA7D1l marker located within the deleted interval clearly indicates the paternal origin of the deletion (fig 6). Studies with markers flanking the deletion interval closely showed that the deletion arose on the paternal chromosome during spermatogenesis by an intrachromosomal mechanism.

To investigate the possibility of somatic mosaicism in BUD's father, we undertook FISH analysis with a BAC from the deletion interval, R-41C23, which flanks the NFI gene on the $3^{\prime}$ side. $^{10}{ }^{14}$ On metaphase chromosomes $(\mathrm{n}=50)$ and interphase nuclei $(n=50)$ of the father, two fluorescent signals were observed, indicating that the chromosomal deletion most probably arose during germ cell maturation.

\section{DISCUSSION}

Previous definition of the $1.4 \mathrm{mbp}$ deletion interval shared by most patients with NFI microdeletion led to the identification and localisation of new LCRs flanking the breakpoint regions. The patient described here belongs to a small subgroup of four patients whose deletion intervals are not bordered by these LCRs. Their telomeric or centromeric

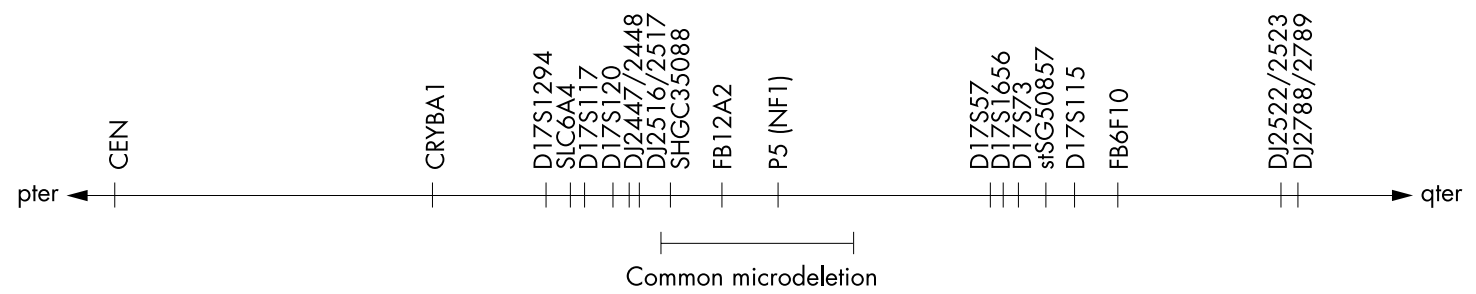

UWA106-3

Common microdeletion

Figure 5 Comparison of large deletions between BUD and four other NF1 patients-UWA106-3,, 151619 UWA155-1 ${ }^{9}$ ID806, ${ }^{17}$ and 3724 A ${ }^{5}$ Relevant plasmid probes (D17S120, D17S57, D17S73, D17S1 15) previously used to detect polymorphic fragments by Southern hybridisation analysis were sequenced and positioned within our clone contig (D17557 (AJ550808); D17573 (AJ550810); D1751 15 (AJ55081 1)). P5 indicates the location of an NF1 CDNA probe, which extends from exon 37 to the end of the coding sequence. ${ }^{20}$ Black bars represent undeleted segments; deleted regions are marked as white bars; possibly deleted uncharacterised regions are given as grey bars. On the right, the respective deletion size is given in Mb. 
breakpoints fall far beyond these boundaries and have not so far been located at the molecular sequence level.

The deletion of the patient BUD described here is more far reaching in telomeric direction and thus we extended our clone and sequence map up to the distal breakpoint. Major portions of this region have been sequenced and assembled as HTGS data by the Human Genome Consortium towards the end our studies and were partially reported recently. ${ }^{21}$ The existence of low copy repeats on BAC 640N20 has prevented an automatic assembly of the adjoining telomeric region. Furthermore, some of the genes between the LCR on BAC R$640 \mathrm{~N} 20$ and the distal breakpoint of patient BUD were not fully annotated and reviewed. We confirmed and fully established the map by FISH experiments with additional BAC and PAC clones that were initially screened to cover the deleted region fully.

Comparative analyses of the human and murine genome sequences enabled us to identify all functional genes that are co-deleted in our patient BUD and may contribute to the clinical phenotype (table 2; fig 3). Thus our patient is not only hemizygous for the 14 genes between the proximal and distal LCRs at 17q11.2, inclusive of the NFI gene, but also for the 27 genes following distally.

The most conspicuous clinical finding in patient BUD is the large number of bilateral spinal neurofibromas. Although spinal neurofibromas develop as isolated asymptomatic tumours in up to $36 \%$ of $\mathrm{NFl}$ patients, ${ }^{22-24}$ multiple symmetrically distributed spinal tumours with clinical implications are very rare in classical NF1 patients. Familial spinal neurofibromatosis (FSNF, MIM 162210) is a rare distinct form of NFl which is characterised by multiple symmetrical spinal nerve root neurofibromas and café au lait spots in all affected family members. ${ }^{25}$ FSNF is associated with NF1 mutations, but is not caused by specific mutations in the NFl gene. ${ }^{26-28}$ It has been suggested that a gene closely linked to the NFI locus is modifying the NFl phenotype in FSNF. ${ }^{28}$ Most probably, the analyses of large deletion patients

\begin{tabular}{|c|c|c|c|c|c|c|c|c|}
\hline $\begin{array}{l}\text { D17S841 } \\
\text { D17S1873 } \\
\text { D17S1841 } \\
\text { D17S975 } \\
\text { D17S1294 } \\
\text { GGAA7D11 } \\
\text { D17S907 } \\
\text { D17S1833 } \\
\text { DI7S1788 } \\
\text { D17S1867 }\end{array}$ & & \begin{tabular}{|r|}
263 \\
122 \\
273 \\
255 \\
258 \\
262 \\
327 \\
158 \\
165 \\
99 \\
\end{tabular} & \begin{tabular}{||r|}
265 \\
134 \\
273 \\
255 \\
254 \\
278 \\
289 \\
158 \\
155 \\
93 \\
\end{tabular} & & $\begin{array}{l}265 \\
140 \\
275 \\
259 \\
254 \\
266 \\
289 \\
164 \\
155 \\
93\end{array}$ & $\begin{array}{l}267 \\
140 \\
275 \\
259 \\
254 \\
266 \\
289 \\
166 \\
155 \\
93\end{array}$ & & \\
\hline $\begin{array}{l}\text { D17S841 } \\
\text { D17S1873 } \\
\text { D17S1841 } \\
\text { D17S975 } \\
\text { D17S1294 } \\
\text { GGAA7D11 } \\
\text { D17S907 } \\
\text { Di7S1833 } \\
\text { DI7S1788 } \\
\text { Di7S1867 }\end{array}$ & \begin{tabular}{|r|}
265 \\
134 \\
273 \\
255 \\
254 \\
278 \\
289 \\
158 \\
155 \\
93 \\
\end{tabular} & $\begin{array}{l}267 \\
140 \\
275 \\
259 \\
254 \\
266 \\
289 \\
166 \\
155 \\
93\end{array}$ & & \begin{tabular}{|r|}
263 \\
122 \\
273 \\
255 \\
258 \\
--- \\
327 \\
158 \\
165 \\
99 \\
\end{tabular} & $\begin{array}{l}267 \\
140 \\
275 \\
259 \\
254 \\
266 \\
289 \\
166 \\
155 \\
93\end{array}$ & & \begin{tabular}{|r|}
265 \\
134 \\
273 \\
255 \\
254 \\
278 \\
289 \\
158 \\
155 \\
93 \\
\end{tabular} & $\begin{array}{l}267 \\
140 \\
275 \\
259 \\
254 \\
266 \\
289 \\
166 \\
155\end{array}$ \\
\hline
\end{tabular}

Figure 6 Haplotype reconstruction of 10 polymorphic markers at $17 q 11.2-12$ in the family of patient BUD. Markers D17S841, DI7S1873, D17S1841, D17S975, and D17S1294 are proximal to the deleted interval, whereas marker GGAA7D1 1 is located in the deleted region. Markers D17S907, D17S1833, D17S1788, and D17S1867 are distal to the telomeric deletion breakpoint. As indicated by an unfilled rectangle, the deletion occurred on the paternal haplotype by an intrachromosomal mechanism. with or without multiple spinal neurofibromas will help to identify this modifying locus. Unfortunately, a search for spinal neurofibromas by MRI has not been done in the patients ID806 and 3724A. Patient UWA155-1 was reported to have one spinal neurofibroma, but it is unknown whether he carries multiple asymptomatic spinal tumours. ${ }^{9}$ However, patient BUD, described here, and patient UWA106-3, ${ }^{9}$ who both have large deletions, developed numerous spinal tumours.

As the number of spinal neurofibromas has not been investigated and quantitated systematically by MRI in patients with LCR mediated NFI microdeletion, we cannot really exclude the genes of the $1.4 \mathrm{mbp}$ interval, but in view of the clinical data reported so far, we suggest that one of the genes distal to the $1.4 \mathrm{mbp}$ interval is implicated in the development of spinal neurofibromas. The most suspicious region for such a locus is the segment between the distal NF1 LCR and the marker FB6F10, which is deleted in both patients and encompasses nine functional genes. Hemizygosity with reduced activity of this modifier gene might predispose patients BUD and UWAl06-3 to the development of multiple spinal neurofibromas. Similarly, a mutation in the modifier locus or a hypomorphic allele of the latter could contribute to the phenotype in patients with FSNF.

Comparing the breakpoint regions in patient BUD at the sequence levels, we did not encounter blocks of low copy repeats around the proximal and distal breakpoints, which could have triggered chromosomal mispairing and unequal homologous recombination during meiosis, as has been observed in patients with $1.4 \mathrm{mbp}$ deletions. ${ }^{11}{ }^{13}$ The proximal breakpoint of BUD is located between position 49221 and 81044 on BAC R-27lKll (AC005562), about 65 kbp centromeric to the proximal $L C R$ of the NFI gene region, which triggers the common $1.4 \mathrm{mbp}$ deletion by recombination with the distal LCR.

A third LCR, which is similar to the regional LCRs at 17q11.2, has been mapped to $17 \mathrm{q} 24 .^{9}$ This distal LCR also contains fragments of the WI-12393 gene. Our mapping of the deletion breakpoint in patient BUD rules out the involvement of another WI-12393 derived LCR, including the one at 17q24.

The comparisons of the long range deletions at $17 \mathrm{q}$ in five different patients including our patient BUD ${ }^{5} 1^{10-17}$ strongly suggest that all deletion breakpoints in these patients are unique and do not coincide with pairs of LCRs situated at the proximal and distal boundaries.

The fact that the distal breakpoints in three patients (UWA106-3, 155-1, and patient $3724 \mathrm{~A}^{59}{ }^{15}$ ) occur within the $1 \mathrm{mbp}$ spanning the ACCNI gene suggests that this region is accessible and transcribed during germ cell development. The distal deletion breakpoint of patient BUD, however, maps far away from the ACCN1 gene to the region of the SLFN genes. In the region around the distal deletion boundary of patient BUD, the human and the mouse genomes again displayed significant discrepancies. Comparing the SLFN genes, we noticed only six human members (SLFNI-6) of the SLFN gene family in the human genome, but 10 murine genes in the otherwise conserved human-mouse interval (fig 4). These findings indicate that the $S L F N$ gene region has been reshaped by multiple breaks and subsequent rearrangements during the evolution of rodents and humans, and that the deletion of patient BUD occurred in this region of recent evolutionary rearrangements.

The proximal deletion breakpoint of patient BUD falls within a $200 \mathrm{kbp}$ large gene-free segment represented by BAC $\mathrm{R}-271 \mathrm{Kll}$. This BAC spans various blocks of low copy repeats-a partial SMS-REP, ${ }^{29}$ a SMURF2 pseudogene fragment, the known WI-12393 related LCR, sequences from chromosome 19-and is followed by the KIAA0160 pseudogene 
fragment covered by the BAC R-778K9. These blocks originate from different genomic locations and have been inserted successively into this region during primate evolution..$^{14}$ We therefore suggest that the proximal breakpoint region again carries intrinsic features which facilitate double stranded breaks and rearrangements.

Approximately $80 \%$ of small intragenic mutations are paternally inherited, ${ }^{30-32}$ whereas meiotically generated microdeletions of the NFI gene region are predominantly of maternal origin. ${ }^{69}{ }^{32} 33$ In 17 of 19 informative cases (89\%) analysed by López-Correa et al, ${ }^{13}$ the deletions occurred on the maternal allele. By contrast, the deletions of patient UWA106-3, ${ }^{15}{ }^{16}$ patient ID806, ${ }^{17}$ patient $96-2,{ }^{12}{ }^{33}$ and patient $\mathrm{PF}^{12}$ that do not end at the proximal and distal NF1 LCRs were of paternal origin, with one exception (patient $3724 \mathrm{~A}^{5}$ ).

To gain insight into the mechanism of recombination, six families containing patients with de novo deletions were previously analysed by haplotyping. ${ }^{33}$ All five deletions on the maternal chromosome occurred by crossovers between nonsister chromatids, whereas the paternal chromosome acquired a de novo deletion by intrachromosomal (sister chromatid) breaks. Interestingly, this latter deletion (patient 96-2) is not bordered by LCRs and is probably restricted to the NFl gene. Likewise, the deletion of patient BUD arose by an intrachromosomal mechanism during male germ cell development. Length variation of deletions across the NF1 locus were also observed in neurofibromas of NFl patients which lost the normal NFl allele by a mitotically acquired interstitial deletion of the normal 17-homologue. ${ }^{34}$ As shown previously, even interstitial deletions between LCRs can occur intrachromosomally during mitosis in somatic cells, probably driven by the recombination between misaligned LCRs in sister chromatids. ${ }^{35}{ }^{36}$ In view of these observations it seems quite reasonable to assume that non-LCR-driven interstitial deletions are primarily generated during mitotic cell division in particular in the course of spermatogenesis.

\section{ACKNOWLEDGEMENTS}

We thank Wolfgang Lasinger, Helene Spöri, Karin Lehmann, and Antje Kollak for expert technical assistance, and Dr Upadhyaya for sending us various $17 \mathrm{q}$-marker plasmids. This research was supported by grants from the Deutsche Forschungsgemeinschaft (HAl082 and KE-724 2-1 (to HK-S) and KFO 113-1 (To DEJ)) and the Deutsche Krebshilfe (No 70-2452/project 790-3075-Ti).

\section{Authors' affiliations}

H Kehrer-Sawatzki, Department of Human Genetics, University of Ulm, Ulm, Germany

S Tinschert, Institute of Medical Genetics, Charité, Humboldt-University, Berlin, Germany

D E Jenne, Department of Neuroimmunology, Max-Planck-Institute of Neurobiology, Martinsried, Germany

Correspondence to: Dr Dieter E. Jenne, Department of Neuroimmunology, Max-Planck-Institute of Neurobiology, Am Klopferspitz 18a, Planegg-Martinsried D-82152, Germany; djenne@neuro.mpg.de

\section{REFERENCES}

1 Friedman JM, Gutmann DH, MacCollin M, et al. Phenotype, natural history and pathogenesis. In: Neurofibromatosis. Baltimore: John Hopkins University and pathogen.

2 Huson SM, Hughes RAC. A pathogenetic and clinical overview. In: The Neurofibromatoses. London: Chapman and Hall, 1994.

3 Heim RA, Kam-Morgan LN, Binnie CG, et al. Distribution of 13 truncating mutations in the neurofibromatosis 1 gene. Hum Mol Genet 1995;4:975-81.

4 Fahsold R, Hoffmeyer S, Mischung C, et al. Minor lesion mutational spectrum of the entire NFI gene does not explain its high mutability but points to a functional domain upstream of the GAP-related domain. Am J Hum Genet 2000;66:790-818.

5 Cnossen MH, van der Est MN, Breuning MH, et al. Deletions spanning the neurofibromatosis type 1 gene: implications for genotype-phenotype correlations in neurofibromatosis type 1? Hum Mutat 1997:9:458-64.
6 Valero MC, Pascual-Castroviejo I, Velasco E, et al. Identification of de novo deletions at the NFl gene: no preferential paternal origin and phenotypic analysis of patients. Hum Genet 1997;99:720-6.

7 Rasmussen SA, Colman SD, Ho VT, et al. Constitutional and mosaic large NFI gene deletions in neurofibromatosis type 1. J Med Genet 1998;35:468-71.

8 Upadhyaya M, Ruggieri M, Maynard J, et al. Gross deletions of the neurofibromatosis type 1 (NF1) gene are predominantly of maternal origin and commonly associated with a learning disability, dysmorphic features and developmental delay. Hum Genet 1998;102:591-7.

9 Dorschner MO, Sybert VP, Weaver M, et al. NF1 microdeletion breakpoints are clustered at flanking repetitive sequences. Hum Mol Genet 2000;9:35-46.

10 Jenne DE, Tinschert S, Stegmann E, et al. A common set of at least 11 functional genes is lost in the majority of NF1 patients with gross deletions. Genomics 2000;66:93-7

11 Jenne DE, Tinschert S, Reimann $\mathrm{H}$, et al. Molecular characterization and gene content of breakpoint boundaries in patients with neurofibromatosis type 1 with 17q11.2 microdeletions. Am J Hum Genet 2001;69:516-27.

12 López-Correa C, Brems H, Lazaro C, et al. Molecular studies in 20 submicroscopic neurofibromatosis type 1 gene deletions. Hum Mutat 1999;14:387-93

13 López-Correa C, Dorschner M, Brems H, et al. Recombination hotspot in NF microdeletion patients. Hum Mol Genet 2001;10:1387-92.

14 Jenne DE, Tinschert S, Dorschner MO, et al. Complete physical map and gene content of the human NF1 tumor suppressor region in man and mouse. Genes Chrom Cancer 2003;37:111-20.

15 Kayes LM, Riccardi VM, Burke W, et al. Large de novo DNA deletion in a patient with sporadic neurofibromatosis 1, mental retardation and dysmorphism. J Med Genet 1992;29:686-90.

16 Kayes LM, Burke W, Riccardi VM, et al. Deletions spanning the neurofibromatosis I gene: identification and phenotype of five patients. Am Hum Genet 1994:54:424-36.

17 Upadhyaya M, Roberts SH, Maynard J, et al. A cytogenetic deletion, del(17) (q1 1.22q21.1), in a patient with sporadic neurofibromatosis type 1 (NF1) associated with dysmorphism and developmental delay. J Med Genet 1996;33:148-52.

18 Moreira ES, Wiltshire TJ, Faulkner G, et al. Limb-girdle muscular dystrophy type $2 G$ is caused by mutations in the gene encoding the sarcomeric protein telethonin. Nat Genet 2000;24:163-6.

19 Shen S, Battersby S, Weaver M, et al. Refined mapping of the human serotonin transporter (SLC6A4) gene within 17q11 adjacent to the CPD and NF1 genes. Eur J Hum Genet 2000;8:75-8.

20 Wallace MR, Marchuk DA, Andersen LB, et al. Type 1 neurofibromatosis gene: identification of a large transcript disrupted in three NFI patients. Science 1990;249:181-6.

21 Van Roy N, Vandesompele J, Berx G, et al. Localization of the 17q breakpoint of a constitutional $1 ; 17$ translocation in a patient with neuroblastoma within a 25-kb segment located between the ACCN1 and TLK2 genes and near the distal breakpoints of two microdeletions in neurofibromatosis type 1 patients. Genes Chromosomes Cancer 2002;35:113-20.

22 Thakkar SD, Feigen U, Mautner VF. Spinal tumours in neurofibromatosis type 1: an MRI study of frequency, multiplicity and variety. Neuroradiology 1999;41:625-9.

23 Poyhonen $M$, Leisti EL, Kytola S, et al. Hereditary spinal neurofibromatosis: a rare form of NF1? J Med Genet 1997;34:184-7.

24 Egelhoff JC, Bates DJ, Ross JS, et al. Spinal MR findings in neurofibromatosis types 1 and 2. Am J Neuroradiol 1992;13:1071-7.

25 Carey JC, Viskochil DH. Neurofibromatosis type 1: A model condition for the study of the molecular basis of variable expressivity in human disorders. Am J Med Genet 1999:89:7-13.

26 Ars E, Kruyer H, Gaona A, et al. A clinical variant of neurofibromatosis type 1. familial spinal neurofibromatosis with a frameshift mutation in the NF1 gene. Am J Hum Genet 1998;62:834-41.

27 Kaufmann D, Muller R, Bartelt B, et al. Spinal neurofibromatosis without cafeau-lait macules in two families with null mutations of the NF1 gene. Am J Hum Genet 2000;69:1395-400.

28 Messiaen L, Riccardi V, Peltonen J, et al. Independent NF1 mutations in two large families with spinal neurofibromatosis. J Med Genet 2003:40:122-6.

29 Park SS, Stankiewicz P, Bi W, et al. Structure and evolution of the SmithMagenis syndrome repeat gene clusters, SMS-REPs. Genome Res 2002;12:729-38

30 Jadayel D, Fain $P$, Upadhyaya $M$, et al. Paternal origin of new mutations in von Recklinghausen neurofibromatosis. Nature 1990:343:558-9.

31 Stephens K, Kayes L, Riccardi VM, et al. Preferential mutation of the neurofibromatosis type 1 gene in paternally derived chromosomes. Hum Genet 1992;88:279-82.

32 Lazaro C, Gaona A, Ainsworth P, et al. Sex differences in mutational rate and mutational mechanism in the NFI gene in neurofibromatosis type 1 patients. Hum Genet 1996:98:696-9.

33 López-Correa C, Brems H, Lazaro C, et al. Unequal meiotic crossover: a frequent cause of NFI microdeletions. Am J Hum Genet 2000;66:1969-74.

34 Colman SD, Williams CA, Wallace MR. Benign neurofibromas in type 1 neurofibromatosis (NF1) show somatic deletions of the NF1 gene. Nat Genet 1995; 11:90-2.

35 Petek E, Jenne DE, Smolle J, et al. Mitotic recombination mediated by the JJAZF1 (KIAA0160) gene causing somatic mosaicism and a new type of constitutional NFI microdeletion in two children of a mosaic female with only few manifestations. J Med Genet 2003;40:520-5.

36 Serra E, Rosenbaum T, Nadal M, et al. Mitotic recombination affects homozygosity for NF1 germline mutations in neurofibromas. Nat Genet $2001 ; 28: 294-6$ 